\title{
Lower Bound of Concurrence for Qubit Systems
}

\author{
Xue-Na Zhu ${ }^{1}$ Shao-Ming Fei ${ }^{2,3}$ \\ ${ }^{1}$ Department of Mathematics, South China University of Technology, Guangzhou 510640, P.R.China \\ ${ }^{2}$ School of Mathematical Sciences, Capital Normal University, Beijing 100048, China \\ ${ }^{3}$ Max-Planck-Institute for Mathematics in the Sciences, 04103 Leipzig, Germany
}

\begin{abstract}
We study the concurrence of four-qubit quantum states and provide analytical lower bounds of concurrence in terms of the monogamy inequality of concurrence for qubit systems. It is shown that these lower bounds are able to improve the existing bounds and detect entanglement better. The approach is generalized to arbitrary qubit systems.
\end{abstract}

\section{Introduction}

Quantum entanglement plays important roles not only in quantum information science [1, 2, 3, 4, but also in many fascinating features in quantum theory, which have puzzled generations of physicists [5]. The fundamental problems in the theory of quantum entanglement is the entanglement detection and quantification. Concurrence [6, 7, 8] is an important measure of quantum entanglement [9, 10, 11, 12, 13, 14]. Different from entanglement of formation which works only for bipartite systems [15, 16], concurrence can be generalized to arbitrary multipartite systems. However, due to the extremizations involved in the computation, analytical formulas of concurrence are available only for twoqubit states [17] and some high dimensional bipartite states with certain symmetries, like isotropic states and Werner states [18] and some special symmetric states [19, 20, 21]. Calculation of concurrence for general quantum states is a formidable task. In particular, quite less has been known about the concurrence of multipartite mixed states.

In [22] analytical lower bounds of concurrence for three-qubit states have been presented based on PPT (positive partial transposition) and realignment operations. Lower 
bounds of concurrence for $M$-qubit states with pure state decompositions given by the generalized Greenberger-Horne-Zeilinger states or the generalized W-state, and for the multipartite $S C$ (Schmidt correlated) states are provided in [23]. Analytical lower bounds of concurrence for general multipartite systems have been discussed in terms of all possible bipartite decompositions in [24].

In this paper, by using a new approach we provide an analytical lower bound of concurrence for general four-qubit mixed quantum states based on monogamy inequalities. The results are generalized to multipartite case. Our lower bounds can improve the previous ones in Refs. 24, 25].

\section{The lower bounds of concurrence for four-qubit systems}

Let $H_{1}, H_{2}, \ldots, H_{N-1}$ and $H_{N}$ be $N$ 2-dimensional vector spaces associated with $N$ quantum systems. The concurrence of a state $|\psi\rangle \in H_{1} \otimes H_{2} \otimes \ldots \otimes H_{N}$ is defined by, up to an $N$ dependent factor $2^{1-\frac{N}{2}}[9,26]$,

$$
C_{12 \ldots N}(|\psi\rangle)=\sqrt{2^{N}-2-\sum_{\alpha} \operatorname{Tr} \rho_{\alpha}^{2}},
$$

where the index $\alpha$ labels all $2^{N}-2$ non-trivial subsystems of the $N$-qubit system and $\rho_{\alpha}$ are the the corresponding reduced density matrices [27].

The concurrence for a mixed state $\rho$ is defined by the convex roof,

$$
C(\rho) \equiv \min _{\left\{p_{i},\left|\psi_{i}\right\rangle\right\}} \sum_{i} p_{i} C\left(\left|\psi_{i}\right\rangle\right)
$$

for all possible pure state decompositions $\rho=\sum_{i} p_{i}\left|\psi_{i}\right\rangle\left\langle\psi_{i}\right|$, where $\left|\psi_{i}\right\rangle \in \mathcal{H}_{1} \otimes \mathcal{H}_{2} \otimes \ldots \otimes \mathcal{H}_{N}$, $0 \leq p_{i} \leq 1$ and $\sum_{i} p_{i}=1$.

For two-qubit case, the concurrence of a two-qubit state $\rho \in H_{1} \otimes H_{2}$ is given by

$$
C(\rho)=\max \left\{\lambda_{1}-\lambda_{2}-\lambda_{3}-\lambda_{4}, 0\right\}
$$

with $\lambda_{1} \geq \lambda_{2} \geq \lambda_{3} \geq \lambda_{4}$ the square roots of the four nonzero eigenvalues of the nonHermitian matrix $\rho \tilde{\rho}, \tilde{\rho}=\left(\sigma_{y} \otimes \sigma_{y}\right) \rho^{*}\left(\sigma_{y} \otimes \sigma_{y}\right)$, where $*$ denotes complex conjugation in the standard basis and $\sigma_{y}$ is the Pauli matrix. 
For convenience, we define bracket $\{a \mid b\}$. One may either take the first element $a$ or the second element $b$ from $\{a \mid b\}$. However, for any given pair $a$ and $b$, once the first (the second) has been taken, then in a formula one always takes the first (the second) element in all the following brackets containing the same two elements $a$ and $b$. Namely, if one takes $\{a \mid b\}=a$, then $\{b \mid a\}=b$; or if one takes $\{a \mid b\}=b$ then $\{b \mid a\}=a$. We set

$$
\begin{gathered}
T_{1}=1+\left\{-\frac{2-x}{2} \mid \frac{2-x}{2}\right\}+\left\{-\frac{2-y}{2} \mid \frac{2-y}{2}\right\}+\left\{-\frac{2-z}{2} \mid \frac{2-z}{2}\right\}, \\
T_{2}=1+\left\{\frac{2-x}{2} \mid-\frac{2-x}{2}\right\}+\left\{-\frac{y}{2} \mid \frac{y}{2}\right\}+\left\{-\frac{z}{2} \mid \frac{z}{2}\right\}, \\
T_{3}=1+\left\{-\frac{x}{2} \mid \frac{x}{2}\right\}+\left\{\frac{2-y}{2} \mid-\frac{2-y}{2}\right\}+\left\{\frac{z}{2} \mid-\frac{z}{2}\right\},
\end{gathered}
$$

and

$$
T_{4}=1+\left\{\frac{x}{2} \mid-\frac{x}{2}\right\}+\left\{\frac{y}{2} \mid-\frac{y}{2}\right\}+\left\{\frac{2-z}{2} \mid-\frac{2-z}{2}\right\}
$$

where $x, y, z \in[0,2] . T_{i}, i=1,2,3,4$ are all greater than or equal to zero for all choices of $x, y$ and $z$ and for each of the two choices allowed by our bracket notation.

For $N$-qubit quantum states the concurrence satisfies the monogamy inequality [28]:

$$
C_{A_{1} \mid A_{2} A_{3} \ldots A_{N}}^{2}(\rho) \geq \sum_{i=2}^{N} C_{A_{1} A_{i}}^{2}(\rho),
$$

where $C_{A_{1} \mid A_{2} A_{3} \ldots A_{N}}(\rho)$ is the concurrence of state $\rho$ under the bipartite bipartition $A_{1}$ and $A_{2} A_{3} \ldots A_{N}$, and $C_{A_{1} A_{i}}(\rho)$ denotes the concurrence of the reduced state $\rho_{A_{1} A_{i}}=$ $\operatorname{Tr}_{A_{2} \ldots A_{i-1} A_{i+1} \ldots A_{N}}(\rho), i=2, \ldots, N$. We denote $C_{i \mid j k l}$ (resp. $C_{i j \mid k l}$ ) the bipartite concurrence under the bipartition $i$ and $j k l$ (resp. $i j$ and $k l$ ), where $i \neq j \neq k \neq l \in\{1,2,3,4\}$.

Theorem 1: For any four-qubit mixed quantum state $\rho$, the concurrence $C(\rho)$ satisfies

$$
C^{2}(\rho) \geq \sum_{i=1}^{3} \sum_{j>i}^{4}\left(T_{i}+T_{j}\right) C_{i j}^{2}(\rho)
$$

[Proof:] The concurrence (1) of a four-qubit pure state $|\psi\rangle$ can be equivalently written as

$$
C_{1234}^{2}(|\psi\rangle)=2 \sum_{i=1}^{4}\left(1-\operatorname{Tr}\left(\rho_{i}^{2}\right)\right)+2\left[\left(1-\operatorname{Tr}^{2}\left(\rho_{12}\right)\right)+\left(1-\operatorname{Tr}^{2}\left(\rho_{13}\right)\right)+\left(1-\operatorname{Tr}^{2}\left(\rho_{14}\right)\right)\right] .
$$

From (10) one has

$$
C_{1234}^{2}(|\psi\rangle)=C_{1 \mid 234}^{2}+C_{2 \mid 134}^{2}+C_{3 \mid 124}^{2}+C_{4 \mid 123}^{2}+C_{12 \mid 34}^{2}+C_{13 \mid 24}^{2}+C_{14 \mid 23}^{2}
$$


The bounds of the terms $C_{12 \mid 34}^{2} C_{13 \mid 24}^{2}$ and $C_{14 \mid 23}^{2}$ in (11) can be further derived. Since $\operatorname{Tr}\left(\rho_{12}^{2}\right)=\operatorname{Tr}\left(\rho_{34}^{2}\right)$ for a four-qubit pure state $|\psi\rangle$, we have

$$
C_{12 \mid 34}^{2}(|\psi\rangle)=x\left(1-\operatorname{Tr}\left(\rho_{12}^{2}\right)\right)+(2-x)\left(1-\operatorname{Tr}\left(\rho_{34}^{2}\right)\right)
$$

where $x \in[0,2]$. Therefore

$$
C_{12 \mid 34}^{2}(|\psi\rangle) \geq x\left[\left(1-\operatorname{Tr}\left(\rho_{12 p}^{2}\right)\right)-\left(1-\operatorname{Tr}\left(\rho_{p}^{2}\right)\right)\right]+(2-x)\left[\left(1-\operatorname{Tr}\left(\rho_{34 q}^{2}\right)\right)-\left(1-\operatorname{Tr}\left(\rho_{q}^{2}\right)\right)\right]
$$

for $p \in\{3,4\}$ and $q \in\{1,2\}$, where the relation $1+\operatorname{Tr}\left(\rho_{A B}^{2}\right) \geq \operatorname{Tr}\left(\rho_{A}^{2}\right)+\operatorname{Tr}\left(\rho_{B}^{2}\right)$ in [29] for bipartite states $\rho_{A B}$ has been used. Four different combinations of choosing $p$ and $q$ in (12) give rise to that $C_{12 \mid 34}^{2}(|\psi\rangle)$ is greater or equal to the following four formulae:

$$
\begin{aligned}
& \frac{x}{2}\left(C_{4 \mid 123}^{2}-C_{3 \mid 124}^{2}\right)+\frac{2-x}{2}\left(C_{2 \mid 124}^{2}-C_{1 \mid 123}^{2}\right), \\
& \frac{x}{2}\left(C_{4 \mid 123}^{2}-C_{3 \mid 124}^{2}\right)+\frac{2-x}{2}\left(C_{1 \mid 123}^{2}-C_{2 \mid 124}^{2}\right), \\
& \frac{x}{2}\left(C_{3 \mid 124}^{2}-C_{4 \mid 123}^{2}\right)+\frac{2-x}{2}\left(C_{2 \mid 124}^{2}-C_{1 \mid 123}^{2}\right)
\end{aligned}
$$

and

$$
\frac{x}{2}\left(C_{3 \mid 124}^{2}-C_{4 \mid 123}^{2}\right)+\frac{2-x}{2}\left(C_{1 \mid 123}^{2}-C_{2 \mid 124}^{2}\right) .
$$

For simplicity, we write

$C_{12 \mid 34}^{2}(|\psi\rangle) \geq \frac{x}{2}\left\{C_{4 \mid 123}^{2}-C_{3 \mid 124}^{2}, C_{3 \mid 124}^{2}-C_{4 \mid 123}^{2}\right\}+\frac{2-x}{2}\left\{C_{2 \mid 124}^{2}-C_{1 \mid 123}^{2}, C_{1 \mid 123}^{2}-C_{2 \mid 124}^{2}\right\}$

where $\{a, b\}$, different from the definitions of $\{\mid\}$, could be either $a$ or $b$.

Similarly we have

$$
C_{13 \mid 24}^{2}(|\psi\rangle) \geq \frac{y}{2}\left\{C_{4 \mid 123}^{2}-C_{2 \mid 134}^{2}, C_{2 \mid 134}^{2}-C_{4 \mid 123}^{2}\right\}+\frac{2-y}{2}\left\{C_{3 \mid 124}^{2}-C_{1 \mid 234}^{2}, C_{1 \mid 234}^{2}-C_{3 \mid 124}^{2}\right\}
$$

and

$$
C_{14 \mid 23}^{2}(|\psi\rangle) \geq \frac{z}{2}\left\{C_{3 \mid 124}^{2}-C_{2 \mid 134}^{2}, C_{2 \mid 134}^{2}-C_{3 \mid 124}^{2}\right\}+\frac{2-z}{2}\left\{C_{4 \mid 123}^{2}-C_{1 \mid 234}^{2}, C_{1 \mid 234}^{2}-C_{4 \mid 123}^{2}\right\}
$$


where $y, z \in[0,2]$. Denote $T_{i j}=T_{i}+T_{j}$, from (11), (13), (14) and (15) we obtain

$$
\begin{aligned}
C_{1234}^{2}(|\psi\rangle) \geq & T_{1} C_{1 \mid 234}^{2}+T_{2} C_{2 \mid 134}^{2}+T_{3} C_{3 \mid 124}^{2}+T_{4} C_{4 \mid 123}^{2} \\
\geq & \left(T_{1}+T_{2}\right) C_{12}^{2}+\left(T_{1}+T_{3}\right) C_{13}^{2}+\left(T_{1}+T_{4}\right) C_{14}^{2} \\
& +\left(T_{2}+T_{3}\right) C_{23}^{2}+\left(T_{2}+T_{4}\right) C_{24}^{2}+\left(T_{3}+T_{4}\right) C_{34}^{2} \\
= & T_{12} C_{12}^{2}+T_{13} C_{13}^{2}+T_{14} C_{14}^{2}+T_{23} C_{23}^{2}+T_{24} C_{24}^{2}+T_{34} C_{34}^{2},
\end{aligned}
$$

where the monogamy inequality (8) has been used in the second inequality.

Let $\rho=\sum_{i} p_{i}|\psi\rangle_{i}\langle\psi|$ be the optimal pure state decomposition of (2) for a four-qubit mixed state $\rho$. For any pure state $\rho^{i}=|\psi\rangle_{i}\langle\psi|$ in the decomposition, we take the same parameters $x, y, z$ and the same way in choosing $\{a \mid b\}$ from $T_{i} \geq 0, i=1,2,3,4$. Denote $\beta$ the index set $\{12,13,14,23,24,34\}$. We have

$$
\begin{aligned}
C^{2}(\rho) & =\left\{\sum_{i} p_{i} C\left(|\psi\rangle_{i}\langle\psi|\right)\right\}^{2} \\
& \geq\left\{\sum_{i} p_{i} \sqrt{\left(\sum_{\beta} T_{\beta} C_{\beta}^{2}\left(\rho^{i}\right)\right)}\right\}^{2} \\
& \geq \sum_{\beta}\left(\sum_{i} p_{i} \sqrt{T_{\beta}} C_{\beta}\left(\rho^{i}\right)\right)^{2} \\
& =\sum_{\beta} T_{\beta}\left(\sum_{i} p_{i} C_{\beta}\left(\rho^{i}\right)\right)^{2} \\
& \geq \sum_{\beta} T_{\beta} C_{\beta}^{2}(\rho),
\end{aligned}
$$

where the relation $\left(\sum_{j}\left(\sum_{i} x_{i j}\right)^{2}\right)^{\frac{1}{2}} \leq \sum_{i}\left(\sum_{j} x_{i j}^{2}\right)^{\frac{1}{2}}$ has been used in the second inequality.

As there are free parameters $x, y$ and $z$, and many ways to choose the elements in $T_{i}$, inequality (91) gives a set of lower bounds of the concurrence. For example, we may fix $x=$ $2, y=0, z=0$ and select appropriate combinations for $T_{i}: T_{1}=1-\frac{2-x}{2}-\frac{2-y}{2}+\frac{2-z}{2}=1$, $T_{2}=1+\frac{2-x}{2}-\frac{y}{2}-\frac{z}{2}=1, T_{3}=1-\frac{x}{2}+\frac{2-y}{2}+\frac{z}{2}=1$, and $T_{4}=1+\frac{x}{2}+\frac{y}{2}-\frac{2-z}{2}=1$. Then we have $C^{2}(\rho) \geq 2\left[C_{12}^{2}+C_{13}^{2}+C_{14}^{2}+C_{23}^{2}+C_{24}^{2}+C_{34}^{2}\right]$.

If we denote $\Lambda$ the set of the lower bound of four-qubit states. In fact, by taking suitable values of $x, y$ and $z$, and selecting appropriate combinations for $T_{i}(i=1, \ldots, 4)$, we have 


$$
\left\{2\left[C_{i_{1} i_{2}}^{2}+C_{i_{1} i_{3}}^{2}+C_{i_{1} i_{4}}^{2}+C_{j_{1} j_{2}}^{2}+C_{j_{1} j_{3}}^{2}+C_{j_{1} j_{4}}^{2}\right], 2\left[C_{12}^{2}+C_{13}^{2}+C_{14}^{2}+C_{23}^{2}+C_{24}^{2}+C_{34}^{2}\right]\right\} \subseteq \Lambda
$$

where $\left\{i_{1}, i_{2}, i_{3}, i_{4}\right\}=\left\{j_{1}, j_{2}, j_{3}, j_{4}\right\}=\{1,2,3,4\}$.

To investigate the strength of the inequality (9), let us consider the following examples.

Example 1. We first consider a simple pure state, $|\psi\rangle=\left|\psi^{+}\right\rangle \otimes|0\rangle \otimes|1\rangle$, where $\left|\psi^{+}\right\rangle=(|00\rangle+|11\rangle) / \sqrt{2}$. From the Eq.(10), we have $C_{1234}(|\psi\rangle)=2$. For this state, one has $C_{12}(|\psi\rangle)=1$ and $C_{13}(|\psi\rangle)=C_{14}(|\psi\rangle)=0$. we take $x=y=z=0$, and $T_{1}=1+\frac{2-x}{2}+\frac{2-y}{2}+\frac{2-z}{2}=4, T_{2}=1-\frac{2-x}{2}-\frac{y}{2}-\frac{z}{2}=0, T_{3}=1-\frac{x}{2}-\frac{2-y}{2}+\frac{z}{2}=0$, and $T_{4}=1+\frac{x}{2}+\frac{y}{2}-\frac{2-z}{2}=0$. Then from the lower bound (9), we have $C_{1234}^{2}(|\psi\rangle) \geq$ $4\left(C_{12}^{2}(|\psi\rangle)+C_{13}^{2}(|\psi\rangle)+C_{14}^{2}(|\psi\rangle)\right)=4$, namely, the state $|\psi\rangle$ saturates the inequality (9). Nevertheless, from the lower bound in [24] one has $C_{1234}(|\psi\rangle) \geq 1$. Hence our bound is better than the one given in [24].

Example 2. Let us consider the one-parameter four-qubit state

$$
\rho=\frac{1-a}{16} I_{16}+a|\psi\rangle\langle\psi|
$$

where $|\psi\rangle=(|0011\rangle+|0101\rangle+|0110\rangle+|1010\rangle) / 2$ and $I_{16}$ is the $16 \times 16$ identity matrix. From (3), we get

$$
C_{12}(\rho)=\max \left\{\frac{1}{4}\left(\sqrt{1+a+a^{2}+2 a \sqrt{1+a}}-\sqrt{1+a+a^{2}-2 a \sqrt{1+a}}-2 \sqrt{1-a}\right), 0\right\} .
$$

From the lower bound in [25] $\rho$ is entangled for $a>0.636364$. While if we take the same $x, y, z$ and $T_{i}$ as in example 1 , from (9) we have that $C(\rho) \geq 2 C_{12}(\rho)>0$ for $a>0.618034$. Hence the bound (91) detects entanglement better.

For multipartite quantum systems, although there are some criteria to detect genuine multipartite entanglement, there is no computable measure in quantifying the multipartite entanglement in general. The example below shows that our lower bound of concurrence for multipartite quantum systems has a tight analytic relations with two-qubit concurrences.

Example 3. We consider the quantum state $\rho=\frac{1-t}{16} I_{16}+t|\psi\rangle\langle\psi|$, where $|\psi\rangle=$ $(|0000\rangle+|0011\rangle+|1100\rangle+|1111\rangle) / 2$. We have

$\rho_{12}=\rho_{34}=\frac{1+t}{4}(|00\rangle\langle 00|+| 11\rangle\langle 11|)+\frac{t}{2}(|00\rangle\langle 11|+| 11\rangle\langle 00|)+\frac{1-t}{4}(|01\rangle\langle 01|+| 10\rangle\langle 10|)$, 
and

$$
\rho_{13}=\rho_{14}=\rho_{23}=\rho_{24}=\frac{1}{4}(|00\rangle\langle 00|+| 01\rangle\langle 01|+| 10\rangle\langle 10|+| 11\rangle\langle 11|) .
$$

Therefore, by using the formula of concurrence for two-qubit states (3), we have

$$
C_{12}(\rho)=C_{34}(\rho)=\max \left\{0, \frac{\sqrt{1+6 t+9 t^{2}}-3(1-t)}{4}\right\}
$$

and $C_{13}=C_{14}=C_{23}=C_{24}=0$. If we take $x=0, y=z=2$, and $T_{1}=1+\frac{2-x}{2}+\frac{2-y}{2}+$ $\frac{2-z}{2}=2, T_{2}=1-\frac{2-x}{2}+\frac{y}{2}+\frac{z}{2}=2, T_{3}=1-\frac{x}{2}-\frac{2-y}{2}-\frac{z}{2}=0$, and $T_{4}=1+\frac{x}{2}-\frac{y}{2}-\frac{2-z}{2}=0$, then from Theorem 1, the lower bound of concurrence is given by:

$$
C^{2}(\rho) \geq 4 C_{12}^{2}(\rho)+2 C_{13}^{2}(\rho)+2 C_{14}^{2}(\rho)+2 C_{23}^{2}(\rho)+2 C_{24}^{2}(\rho) .
$$

From Fig. 1, we see that the lower bound can detect entanglement of $\rho$ when $t>1 / 3$.

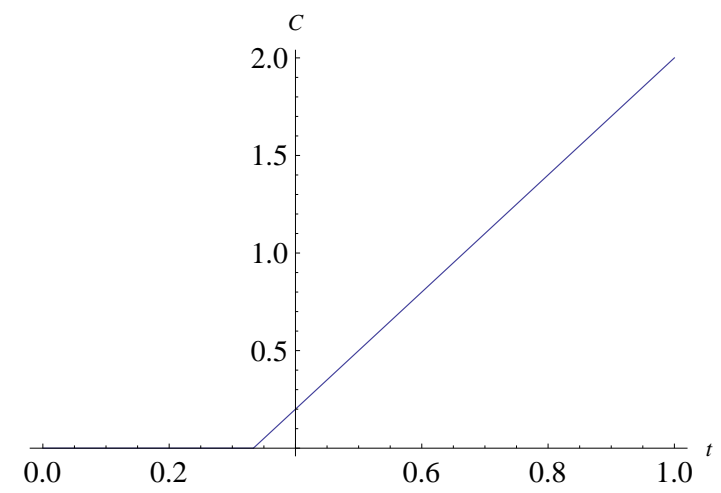

Fig. 1. The lower bound concurrence of $\rho$ for $0 \leq t \leq 1$.

\section{3. the lower bounds of concurrence for arbitrary qubit sys- tems}

Now we generalize our results to $N$-qubit systems. For a given $N$-qubit state $|\psi\rangle \in$ $H_{1} \otimes H_{2} \otimes \ldots \otimes H_{N}$, the concurrence (11) has the form

$$
C_{12 \ldots N}^{2}(|\psi\rangle)=\sum_{\vec{j}} \frac{1}{2} C_{\vec{j} \mid R(\vec{j})}^{2},
$$

where $\vec{j}=\left\{j_{1}, j_{2}, \ldots, j_{r}\right\} \subseteq\{1,2, \ldots, N\}$ are all the possible integer strings, $j_{1}<j_{2}<\ldots<$ $j_{r}$, such that $\vec{j} \cup R(\vec{j})=\{1,2, \ldots, N\}$, i.e. $R(\vec{j})=\{1,2, \ldots, N\} \backslash \vec{j}$. 
Similar to the four-qubit case, taking into account that $1+\operatorname{Tr} \rho_{A B}^{2} \geq \operatorname{Tr}\left(\rho_{A}^{2}\right)+\operatorname{Tr}\left(\rho_{B}^{2}\right)$, we can prove the following corollary:

Corollary 1: For any N-qubit pure state $|\psi\rangle$, the concurrence $C_{\vec{j} \mid R(\vec{j})}$ satisfies $C_{\vec{j} \mid R(\vec{j})}^{2} \geq \frac{1}{2}\left\{x C_{j_{t} \mid R\left(\left\{j_{t}\right\}\right)}^{2}+(2-x) C_{j_{s} \mid R\left(\left\{j_{s}\right\}\right)}^{2}-x \sum_{j_{p}} C_{j_{p} \mid R\left(\left\{j_{p}\right\}\right)}^{2}-(2-x) \sum_{j_{q}} C_{j_{q} \mid R\left(\left\{j_{q}\right\}\right)}^{2}\right\}$,

where: $j_{t} \in R(\vec{j}), j_{s} \in \vec{j}, j_{p} \in R(\vec{j}) \backslash\left\{j_{t}\right\}, j_{q} \in \vec{j} \backslash\left\{j_{s}\right\}$ and $x \in[0,2]$.

From the corollary, in terms of the monogamy relation (8) , for any N-qubit $(N \geq 4)$ mixed quantum state $\rho$, there are some fixed numbers numbers $F_{i} \geq 0, i=1, \ldots, N$, which depend on parameters like $T_{i}$ in Theorem 1 and the concurrence $C(\rho)$ satisfies

$$
C^{2}(\rho) \geq \sum_{i=1}^{N-1} \sum_{j>i}^{N}\left(F_{i}+F_{j}\right) C_{i j}^{2}
$$

\section{Conclusion}

In summary, we have proposed a new approach in constructing hierarchy of lower bounds of concurrence for four-qubit mixed states in terms of the monogamy inequality of concurrence. The lower bounds may be used to improve the previous lower bounds of concurrence and can detect better quantum entanglement. Besides, our approach can be generalized to $N$-qubit systems to obtain the lower bound of the concurrence for $N$-qubit states.

\section{References}

[1] M. A. Nielsen and I. L. Chuang, Quantum Computation and Quantum Information (Cambridge University Press, Cambridge, 2000).

[2] A. Einstein, B. Podolsky, and N. Rosen, Phys. Rev. 47, 777 (1935).

[3] A. Osterloh, L. Amico, G. Falci, and R. Fazio, Nature (London) 416, 608 (2002).

[4] R. F. Werner, Phys. Rev. A 40, 4277 (1989).

[5] L. Amico, R. Fazio, A. Osterloh, and V. Vedral, Rev. Mod. Phys. 80, 517 (2008).

[6] W. K. Wootters, Phys. Rev. Lett. 80, 2245 (1998). 
[7] A. Uhlmann, Phys. Rev. A 62, 032307 (2000).

[8] P. Rungta, V. Bužek, C. M. Caves, M. Hillery and G. J. Milburn, Phys. Rev. A 64, 042315 (2001).

[9] F. Mintert, M. Kuś, and A. Buchleitner, Phys. Rev. Lett. 92, 167902 (2004).

[10] K. Chen, S. Albeverio, and S. M. Fei, Phys. Rev. Lett. 95, 040504 (2005).

[11] H. P. Breuer, J. Phys. A: Math. Gen. 39, 11847 (2006).

[12] H. P. Breuer, Phys. Rev. Lett. 97, 080501 (2006).

[13] J. I. de Vicente, Phys. Rev. A 75, 052320 (2007).

[14] C. J. Zhang, Y. S. Zhang, S. Zhang, and G. C. Guo. Phys. Rev. A 76, 012334 (2007).

[15] C. H. Bennett, H. J. Bernstein, S. Popescu, and B. Schumacher, Phys. Rev. A 53, 2046 (1996).

[16] C. H. Bennett, D.P. DiVincenzo, J. A. Smolin, and W. K. Wootters, Phys. Rev. A 54, 3824 (1996).

[17] W. K. Wootters, Phys. Rev. Lett. 80, 2245 (1998).

[18] K. G. H. Vollbrecht and R. F. Werner, Phys. Rev. A 64, 062307 (2001).

[19] P. Rungta and C. M. Caves, Phys. Rev. A 67, 012307 (2003).

[20] K. Chen, S. Albeverio, and S.M. Fei, Rep. Math. Phys. 58, 325 (2006).

[21] S. M. Fei, Z. X. Wang, and H. Zhao, Phys. Lett. A 329, 414 (2004).

[22] X. H. Gao, S. M. Fei, and K. Wu, Phys. Rev. A 74, 050303 (2006).

[23] X. H. Gao and S. M. Fei, Eur. Phys. J. Special Topics 159, (71) (2008).

[24] X. H. Gao , A. Sergio, K. Chen, S. M. Fei, and X. Q. Li-Jost. Front.Comput.Sci. China 2008,2(2):114-128.

[25] Z. H. Chen, Z. H. Ma, J. L. Chen, and S. Severini, Phys. Rev. A 85, 062320 (2012). 
[26] A. R. R. Carvalho, F. Mintert, and A. Buchleitner, Phys. Rev. Lett. 93, 230501 (2004).

[27] L. Aolita and F. Mintert, Phys. Rev. Lett. 97, 050501 (2006).

[28] T. J. Osborne and F. Verstraete, Phys. Rev. Lett. 96, 220503 (2006).

[29] C. J. Zhang, Y. X. Gong, Y. S. Zhang, and G. C. Guo, Rev. A 78, 042308 (2008). 